\title{
Realizing the Potential for Real Surface and Nanoparticle Analysis with an SEM
}

\author{
Edward D Boyes
}

\section{DuPont Company, CR\&D, Wilmington, DE, 19880-0356, USA}

A primary advantage of the SEM is the convenient bulk specimen form, including unthinned silicon wafer pieces, and nm-scale analyses on the surfaces of unmodified sub-micron particles. No meaningful sample : no useful data. Historically there have been rather low performance expectations for this type of instrument; based in part on a limited, or actually incorrect, understanding of the critical beam-specimen interaction physics. "The problem is not just what we don't know, but what we do know that ain't so" (Mark Twain). The new proposition [1] is that at least down to $0.4 \mathrm{~nm}$, the spatial resolution of secondary electron imaging at low beam energies (where most of the interest and action is these days) is limited primarily by the instrument probe size; convoluted to a greater or lesser extent with the signal generation range in the sample. Lower is generally smaller and better. And may remove the need to apply a conductive coating; allowing direct surface imaging.

Pressing practical application needs, especially in the semiconductor industry and myriad nanotechnology applications, have driven a revolution in SEM instrumentation and methods with wide utility across science and industry. Some of these are true nanotechnology (deliberate build up from the bottom), and some of them kind of "incidental nanotechnology" (nano-features achieved more serendipitously and/or developed before this regime was attributed to a separate discipline). For example much heterogeneous catalysis was nano before nano was nano.

It is now an entirely different ball game in SEM with image resolutions down to $\sim 1 \mathrm{~nm}$ at $1 \mathrm{kV}$, $<100 \mathrm{~nm}$ lateral chemical nanoanalysis and thin film detection sensitivities down to $\sim 1 \mathrm{~nm}$ (the latter limited by the $\mathrm{P} / \mathrm{B}$ of the widely employed conventional EDS detectors) on bulk samples. The new capabilities will be illustrated with examples of sub-micron particle surface analyses which have generated new information and new insights into materials surface structure/property relationships. The current action is mostly at low beam energies of $\sim 1 \mathrm{kV}$ for imaging and $\sim 5 \mathrm{kV}$ for analysis; now that some of the major instrumental limitations (many of them once considered fundamental) have been overcome or circumvented. For example, it has been possible to develop new operating modes of a special condenser-objective lens to obtain what are probably the lowest natural aberration coefficients ever achieved in electron optics (Cs $\sim 0.1 \mathrm{~mm}, \mathrm{Cc} \sim 0.2 \mathrm{~mm})$. Because of the much reduced lens excitation requirements, this turns out to be practical for low voltage SEM in ways which probably are not for TEM applications. The next step is active aberration correction, especially at low voltages for $\mathrm{Cc}$ and $\Delta \mathrm{E}[2]$.

One down side is that at lower voltages and higher resolutions the trademark depth of field of the SEM is more limited. This disappears disproportionately $\left(F\left(\alpha^{2}\right)\right)$ with resolution improvements $(\mathrm{F}(\alpha)$ ) and beam energy reductions. A second one (and there may well be others) is the surface sensitivity of the analysis. While in some applications this is clearly a major advantage, in others this can be seen as a problem, especially where it interferes with subsurface analysis. However, it has been shown previously [3] that the metrics for the analysis of fine scale geometries, both laterally and vertically, swamp any effects of reduced correction accuracy [4] under low voltage 
analysis conditions. Low voltage analysis can provide valuable new information and in this paper we argue to use the surface sensitivity to advantage. It is however probably true [4] that it should not be used to analyze multi-micron sized features unless it is necessary; such as for superior light element analysis.

The greatly increased surface sensitivity of analysis can of course have a downside as well as an upside. For example, oxidized or otherwise contaminated sample surfaces can give erroneous results, as they can in most surface analyses. Some of this may be due to transfer to the microscope through air, and as for many other surface tools it may be necessary to add a controlled atmosphere transfer device. Cross-section sample preparation may also require extra care.

There are inherent advantages in operating over an extended magnification range with both nmscale imaging and sub-micron (down to a nanometer or so) elemental analyses of representative bulk real world samples. This technology also forms the basis of semiconductor line width measurement and defect review tools, and the fundamental surface sensitive attributes underpinning these applications are much more universally applicable across many fields.

This amounts to a reinvention of the SEM in a different form, and certainly with much more ambitious and useful goals. The wide area bulk sample form is simple and quick to prepare; or just to select, as the case may be. It is also inherently robust and generally representative with direct access to native surfaces in plan view or simple cross-sections. From sub-micron particles, the 3D representation of surfaces in SEM data is especially informative; both in its own right and to guide atomic resolution STEM experiments. The chemical analysis of surface particles and thin films on larger substrates (micron particles or bulk wafers etc) has a demonstrated sensitivity of $\sim 1 \mathrm{~nm}$ for alumina or carbon. This is a combination of the effects of low beam voltages and beam penetration together with the EDS detector characteristics. New technology spectrometers, with improved energy resolution and peak to background ratios, should reduce the sensitivity limits closer to $0.1 \mathrm{~nm}$; ie. $<1 \mathrm{ML}$. At which point, of course, surface preservation becomes an even bigger, but not entirely new, issue. Even in clean oil-free vacuum systems, carbon contamination is often seen.

Even at the present level, the modified SEM is a powerful analytical tool for surface analysis; especially for practical problem solutions requiring lateral spatial resolution for the analysis of heterogeneous real world and fabricated nanotechnology samples. In the process, new surface topographies - the particle interface with the outside world - have been discovered and analyzed chemically and crystallographically. The latter by classical $18^{\text {th }}$ century methods of symmetry and interplanar angle measurement applied on a microscopic or nanoscopic scale. Of course, fully to benefit from these advances we will have to be more careful in handling specimens for analysis, including by ESEM preservative methods and others avoiding conductive coatings, as well as in any preparation which may be required, and in transfer into the microscope. In summary, SEM is entering a new and arguably more scientifically useful era. It is no longer a mature technology.

\section{References}

1. E D Boyes, Microscopy and Microanalysis, 11/2 (2005) 2112

2. M Haider et al, Nature, 383 (1998) 768

3. E D Boyes, Mikrochimica Acta, 138 (2002) 225

4. P J Statham, Microscopy Today, (2006) 